\title{
Physical activity levels and social interactions of preschoolers with and without disabilities
}

\author{
Seokheon Kang, So-Yeun Kim* , Lauriece L. Zittel, Marilyn Looney \\ Department of Kinesiology and Physical Education, College of Education, Northern Illinois University, DeKalb, IL, USA
}

This study examined differences of physical activity levels and social interactions among preschoolers with/without disabilities and those at risk for a developmental delay (DD). Sixty preschoolers (20 in each group) were film during their designated large gross motor time. Their physical activity levels and social interaction were examined using the physical activity level screening. Regarding physical activity levels, the result of a two-way analysis of variance indicated significant main effects for groups, $F(2,54)=4.192, P=0.02$; preschoolers at risk for a DD were more active than preschoolers without disabilities, $P=0.02$. For social interaction, a significant main effect for groups, $F(2,54)=8.994$,
$P<0.001$ was found; Preschoolers at risk for a DD were more interactive than preschoolers with/without disabilities, $P=0.001$ and $P=0.002$, respectively. There were no significant gender differences across three groups on physical activity levels and social interactions. Although preschoolers at risk for a DD was more active and interactive than the other groups, preschoolers across three groups engaged in light physical activity levels, and had limited social interactions with peers.

Keywords: Physical activity, Unstructured playtime, Preschoolers, Disability

\section{INTRODUCTION}

Physical activity plays an important role in children's emotional, social, motor, and physical development as a whole (Roberts et al., 2012). Physical activity enables children to alleviate the risk of having cardiovascular disease while improving body composition and physical fitness (Vasconcellos et al., 2014). Moreover, physical activity can provide children with valuable time to be physically and socially active with peers (Ramstetter et al., 2010).

According to the guideline by the National Association for Sport and Physical Education in 2009, preschoolers should participate in a minimum of $120 \mathrm{~min}$ of structured and unstructured physical activity (60 min each) every day (Beets et al., 2011). During physical activity time, preschoolers should also enjoy and actively participate in physical activity, not being sedentary for more than 60 min except when sleeping.

There have been inconsistence findings whether preschoolers meet the national guideline. Beets et al. (2011) reported that the prevalence of meeting the national guideline for physical activity among United States preschoolers were various substantially across studies. Tucker (2008) concluded that health promotion efforts were need for increasing physical activity levels of preschoolers since only $54 \%$ of preschoolers were sufficiently physically active based on the results of his systematic review research.

Childcare centers or early childhood programs can be a great setting to promote physical activity levels of preschoolers since they provide designated large gross motor time. Limited study has been conducted to examine physical activity levels of preschoolers during their designated large gross motor time. Understanding preschoolers' physical activity behaviors during designated large gross motor time is critical to develop effective physical activity/exercise intervention programs since participation in regular physical activity plays a crucial role in the prevention and treatment of obesity (Hill and Wyatt, 2005).

The purpose of this study, therefore, was to examine preschoolers' physical activity levels accompanying social interactions
${ }^{*}$ Corresponding author: So-Yeun Kim (iD https://orcid.org/0000-0001-5779-7450 Department of Kinesiology and Physical Education, College of Education, Northern Illinois University, 1425 W. Lincoln Hwy., DeKalb, IL 60115, USA E-mail: soyeunkim@niu.edu

Received: September 21, 2018 / Accepted: December 18, 2018
This is an Open Access article distributed under the terms of the Creative Commons Attribution Non-Commercial License (http://creativecommons.org/licenses/by-nc/4.0/) which permits unrestricted non-commercial use, distribution, and reproduction in any medium, provided the original work is properly cited. 
during their unstructured large gross more time were examined. Specific research questions include (a) Are there any significant group (preschoolers with/without a disability and those at risk for a developmental delay [DD]) and gender differences in physical activity levels during designated large gross motor time? and (b) Are there any significant group and gender differences in social interactions during designated large gross motor time?

\section{MATERIALS AND METHODS}

\section{Participants}

A total of 60 preschoolers (30 male and 30 female), aged from 36 to 72 months, participated in this study after receiving the Institutional Review Board approval (\#HS16-0093) from the Northern Illinois University in the United States. Among the participants, there were 20 preschoolers with a DD, 20 preschoolers at risk for a DD, and 20 preschoolers without a disability from two early childhood programs. The mean ages of preschoolers with a disability, those without disability, and those at risk for a DD were 48.7 months (standard deviation [SD], 7.2), 48.7 months (SD, 5.1), and 58.3 months (SD, 7.3), respectively.

\section{Instruments}

The physical activity level screening (PALS) (Park et al., 2017), a systematic observation tool, was used to measure physical activity level and social interactions of participants during designated large gross motor time. The PALS uses 15-sec momentary time sampling. When using the PALS, physical activity levels are measured in three categories including none, light, and moderate/vigorous physical activity (MVPA) while social interaction of preschoolers was measured in three categories including play alone, parallel play and interactional play. The validity evidence of the PALS was established for physical activity level of preschoolers with a DD/or a disability using actical accelerometers, $r=0.67$, $P<0.01$ (95\% confidence interval: 0.5-0.9) (Park et al., 2017). Content validity evidence of social interaction were validated in previous study by Hestenes and Carroll (2000). Both physical activity levels and social interactions were recorded numerically (e.g., none $=0$, light $=1, \mathrm{MVPA}=2$; play alone $=0$, parallel play $=$ 1 , interactional play $=2$ ).

\section{Procedures}

Participants were filmed during their 25-min designated/unstructured large gross motor time on playgrounds. When the data collection was done, two graduate students received trainings on use of the PALS from two faculty members. When the training was completed, two raters coded three videos independently to examine interrater reliability of physical activity levels and social interactions of PALS. One week later, raters coded each video again to examine intrarater reliability. The scores of intra- and interrater reliability for both raters from three subjects met the acceptable proportion of agreement $(\geq 90 \%)$.

\section{Actual coding}

Two raters watched video clips independently and assessed each preschooler's physical activity levels and social interactions using the PALS; Rater 1 coded all participants whereas rater 2 coded randomly selected 30 participants in order to check interrater reliability of rater 1 . If the participant was not seen on the film in that 15-sec recording time, the raters described it as "N/A" for that section. If the percentage of "N/A" was more than $10 \%$, these data were not used in this study.

The average proportions of agreement for intrarater reliability by rater 1 for physical activity levels, physical activity choices, and social interactions were 0.92 (SD, 4.2), 0.94 (SD, 3.9), and 0.95 (SD, 3.2), respectively. The means of modified Cohen kappa coefficient for intrarater reliability from rater1 were 0.88 (SD, 6.2), 0.91 (SD, 5.8), and 0.92 (SD, 5.3). The average proportions of agreement for intrarater reliability by rater 2 for physical activity levels and social interactions were 0.93 (SD, 4.9) and 0.92 (SD, 7.8), respectively. The means of modified Cohen kappa coefficient for intrarater reliability from rater 2 were 0.89 (SD, 7.3) and 0.88 (SD, 11.2). The average proportions of agreement for interrater reliability between rater1 and rater 2 for physical activity levels and social interactions were 0.83 (SD, 5.0) and 0.84 (SD, 7.4), respectively. The means of modified Cohen kappa coefficient for interrater reliability were $0.76(\mathrm{SD}, 7.4)$ and $0.76(\mathrm{SD}, 11.0)$, respectively.

\section{Statistical analyses}

The data analysis was conducted for the data of 60 participants from the first coding by rater 1 . The means and standard deviations of physical activity levels and social interactions were calculated for preschoolers with/without a disability and those at risk for a DD, and calculated for gender across the three groups of preschoolers. A two-way analysis of variance (ANOVA) was used to examine differences between means of physical activity levels and social interactions for group by gender. The significance level was set at 0.05 . A Tukey honestly significant difference test was conducted after a significant group effect. Each individual's data point 
used in the analysis was his/her average score over the 25-min observation time for physical activity levels and social interactions separately.

\section{RESULTS}

The average percentage of N/A for 60 participants was 4.14 . Among 60 participants, a total of 54 participants had at least one N/A (range, 1-10). The means of physical activity levels for female preschoolers with/without a disability and those at risk for a DD were 0.97 (SD, 0.41), 0.74 (SD, 0.16), and 1.06 (SD, 0.18), respectively. The means of physical activity level for male preschoolers with/without a disability and those at risk for a DD were 0.83 (SD, 0.15), 0.84 (SD, 0.13), and 0.92 (SD, 0.18), respectively. The means of social interactions for female preschoolers with/without a disability and those at risk for a DD were 0.79 (SD, 0.19), 0.69 (SD, 0.32), and 1.05 (SD, 0.22), respectively. The means of social interactions for male preschoolers with/without a disability and those at risk for a DD were 0.62 (SD, 0.21), and 0.73 (SD, 0.30), and 0.95 (SD, 0.24), respectively. Table 1 shows the average percentage of each category in physical activity levels and social interactions among participants based on the group and gender.

The result of a two-way ANOVA indicated that there were significant main effects for groups, $F(2,54)=4.192, P=0.02$. There was no significant difference between preschoolers with a disability and those at risk for a $\mathrm{DD}(P=0.40)$, but preschoolers at risk for a DD were significantly more active than preschoolers without a disability $(P=0.02)$. No significant difference was found between preschoolers with and without a disability $(P=0.26)$. There was no significant main effect for gender, $F(1,54)=0.959, P=0.33$, and no significant interaction, $F(2,54)=1.812, P=0.17$.

Table 1. The average percentages of physical activity levels and social interactions

\begin{tabular}{|c|c|c|c|c|c|c|}
\hline \multirow{2}{*}{ Variable } & \multicolumn{2}{|c|}{ Without a disability } & \multicolumn{2}{|c|}{ At risk for a DD } & \multicolumn{2}{|c|}{ With a disability } \\
\hline & Boys & Girls & Boys & Girls & Boys & Girls \\
\hline \multicolumn{7}{|c|}{ Physical activity levels } \\
\hline None & 25.5 & 27.0 & 18.2 & 12.6 & 21.0 & 17.0 \\
\hline Light & 63.2 & 64.9 & 66.7 & 63.4 & 67.1 & 61.1 \\
\hline MVPA & 8.6 & 6.1 & 9.9 & 18.0 & 6.7 & 18.2 \\
\hline \multicolumn{7}{|l|}{ Social interactions } \\
\hline Play alone & 34.5 & 34.9 & 27.7 & 17.6 & 39.1 & 27.0 \\
\hline Parallel play & 54.0 & 57.2 & 52.6 & 57.3 & 51.4 & 61.5 \\
\hline Interactional play & 9.1 & 5.2 & 15.6 & 21.9 & 4.8 & 8.9 \\
\hline
\end{tabular}

$\mathrm{DD}$, developmental delay; MVPA, moderate/vigorous physical activity.
Regarding the effect of groups and gender for social interaction, results from the two-way ANOVA indicated a significant main effect for group, $F(2,54)=8.994, P<0.001$. Preschoolers at risk for a DD were significantly more interactive than preschoolers with a disability $(P=0.001)$ and without a disability $(P=0.002)$. No significant difference was found between preschoolers with and without a disability $(P=1.0)$. There was no significant main effect for gender, $F(2,54)=1.358, P=0.25$, and no significant interaction, $F(2,54)=0.955, P=0.39$.

\section{DISCUSSION}

The purpose of this study was to examine preschoolers' physical activity levels and types of social interactions during designated large gross motor time. The results of this study indicated that there were significant differences in the physical activity levels and social interactions between preschoolers at risk for a DD and with/without a disability. However, there were no significant differences in physical activity levels and social interactions between genders.

There was no significant difference in physical activity levels between preschoolers with and without a disability in this study. Similar results were found by Schenkelberg et al. (2017); No significant differences were found between preschoolers with and without autism spectrum disorder during free play and in a structured activity setting. Different ratios between teachers and preschoolers for each group might affect physical activity levels of preschoolers. Especially the class of preschoolers with a disability had a higher teacher-child ratio. For the given number of participants (16-20 preschoolers) on the playground, there were approximately eight teachers for preschoolers with a disability whereas the class of preschoolers without a disability and those at risk for a DD had only two to three teachers. Furthermore, the investigator found that teachers in the class of preschoolers with a disability were more likely to encourage their students to be physically active by holding hands with students to walk around the playground and suggesting riding on a see-saw or swing. Previous studies have announced that the encouragement from a staff/ teacher was positively related to preschoolers' physical activity level (Gubbels et al., 2011; Tonge et al., 2017).

In the current study, preschoolers at risk for a DD were significantly more active than those without a disability whereas no difference was found between preschoolers with a disability and those at risk for a DD. A possible explanation could be related to environmental factors. The playground environments (such as the 
size of the playground and the accessibility to the equipment) were different between preschoolers without a disability and those at risk for a DD. The data from the previous studies concluded that the differences in facilities, seasons, gender, and location were highly associated with the physical activity levels of preschoolers (Baranowski et al., 1993; Gubbels, 2014).

In addition, preschoolers' physical activity levels could be influenced by age. Bult et al. (2013) found that preschoolers ages 4 through 5 years were more actively engaged in activities with a higher intensity score than preschoolers ages 2 through 3 years $(P<0.001)$. Regarding gender differences in physical activity levels, there were no significant differences for all groups. The results of this study are similar with previous studies examining the differences in physical activity levels between male and female preschoolers. According to the study by Cardon and De Bourdeaudhuij (2008), there were no significant gender differences of daily physical activity levels in preschoolers.

Regardless of groups and gender, low physical activity levels have been found in preschoolers. Most of the preschoolers spent majority of their time in sedentary or light activities. The average percentage of MVPA for all groups and gender in this study was $11.3 \%$. Previous studies reported that preschoolers engaged in MVPA for less than $5 \%$ of their playtime (Brown et al., 2006; Cardon and De Bourdeaudhuij, 2008; Pate et al., 2008). These studies support a need to increase the preschoolers' physical activity levels through an appropriate intervention and engagement from teachers.

The results of this study showed that there was no significant difference in social interactions between preschoolers with and without a disability. However, preschoolers at risk for a DD were more interactive with peers than preschoolers with and without a disability. One of the explanations for these significant differences between groups may be the participant's age. The mean ages of preschoolers with, without a disability and those at risk for a DD were 48.65, 48.65, and 58.3 months respectively. According to Barbu et al. (2011), younger preschoolers 2 to 4 years of age were less likely to play with peers and play more with adults rather than older preschoolers aged 4 to 6 years old.

In regards to gender differences, the differences in social interactions between male and female preschoolers for all groups were not significant. A similar result was also reported in the study by Woods et al. (2015), social interaction of younger children (6 years of age) was not significantly different between boys and girls during recess time.

In conclusion, the preschoolers across three groups engaged in light physical activity levels, and had limited social interactions with peers during designated large gross motor time regardless of gender although preschoolers at risk for DD were significantly more active and interactive with their peers compared to other groups. These results point to a need for more structured large gross motor time in preschool settings should be considered to promote physical activity and social interactions in preschoolers with all abilities.

\section{CONFLICT OF INTEREST}

No potential conflict of interest relevant to this article was reported.

\section{REFERENCES}

Baranowski T, Thompson WO, DuRant RH, Baranowski J, Puhl J. Observations on physical activity in physical locations: age, gender, ethnicity, and month effects. Res Q Exerc Sport 1993;64:127-133.

Barbu S, Cabanes G, Le Maner-Idrissi G. Boys and girls on the playground: sex differences in social development are not stable across early childhood. PLoS One 2011;6:e16407.

Beets MW, Bornstein D, Dowda M, Pate RR. Compliance with national guidelines for physical activity in U.S. preschoolers: measurement and interpretation. Pediatrics 2011;127:658-664.

Brown WH, Pfeiffer KA, Mclver KL, Dowda M, Almeida MJ, Pate RR. Assessing preschool children's physical activity: the Observational System for Recording Physical Activity in children-preschool version. Res Q Exerc Sport 2006;77:167-176.

Bult MK, Verschuren O, Kertoy MK, Lindeman E, Jongmans MJ, Ketelaar M. Psychometric evaluation of the dutch version of the assessment of preschool children's participation (APCP): construct validity and test-retest reliability. Phys Occup Ther Pediatr 2013;33:372-383.

Cardon GM, De Bourdeaudhuij IM. Are preschool children active enough? Objectively measured physical activity levels. Res Q Exerc Sport 2008; 79:326-332.

Gubbels JS. Physical activity in childcare settings: the role of the environment. Sci Sports 2014;29 Suppl:41-42.

Gubbels JS, Kremers SP, van Kann DH, Stafleu A, Candel MJ, Dagnelie PC, Thijs C, de Vries NK. Interaction between physical environment, social environment, and child characteristics in determining physical activity at child care. Health Psychol 2011;30:84-90.

Hestenes LL, Carroll DE. The play interactions of young children with and without disabilities: Individual and environmental influences. Early Child Res Q 2000;15:229-246. 
Hill JO, Wyatt HR. Role of physical activity in preventing and treating obesity. J Appl Physiol (1985) 2005;99:765-770.

Park C, Kim, SY, Zittel LL, Looney M. Validity and reliability evidence of the physical activity level screening for preschoolers with developmental delays and/or disabilities. J Exerc Rehabil 2017;13:210-213.

Pate RR, McIver K, Dowda M, Brown WH, Addy C. Directly observed physical activity levels in preschool children. J Sch Health 2008;78:438444.

Ramstetter CL, Murray R, Garner AS. The crucial role of recess in schools. J Sch Health 2010;80:517-526.

Roberts SJ, Fairclough SJ, Ridgers ND, Porteous C. An observational assessment of physical activity levels and social behaviour during elementary school recess. Health Educ J 2012;72:254-262.

Schenkelberg MA, Rosenkranz RR, Milliken GA, Menear K, Dzewaltowski DA. Implications of social groups on sedentary behavior of children with autism: a pilot study. J Autism Dev Disord 2017;47:1223-1230.

Tonge KL, Jones RA, Hagenbuchner M, Nguyen TV, Okely AD. Educator engagement and interaction and children's physical activity in early childhood education and care settings: an observational study protocol. BMJ Open 2017;7:e014423.

Tucker P. The physical activity levels of preschool-aged children: a systematic review. Early Child Res Q 2008;23:547-558.

Vasconcellos F, Seabra A, Katzmarzyk PT, Kraemer-Aguiar LG, Bouskela E, Farinatti P. Physical activity in overweight and obese adolescents: systematic review of the effects on physical fitness components and cardiovascular risk factors. Sports Med 2014;44:1139-1152.

Woods AM, Graber KC, Daum DN, Gentry C. Young school children's recess physical activity: movement patterns and preferences. J Teach Phys Educ 2015;34:496-516. 